\title{
Matrix product variational formulation for lattice gauge theory
}

\author{
Takanori Sugihara \\ RIKEN BNL Research Center, Brookhaven National Laboratory, Upton, New York 11973, USA
}

\begin{abstract}
For hamiltonian lattice gauge theory, we introduce the matrix product anzats inspired from density matrix renormalization group. In this method, wavefunction of the target state is assumed to be a product of finite matrices. As a result, the energy becomes a simple function of the matrices, which can be evaluated using a computer. The minimum of the energy function corresponds to the vacuum state. We show that the $S=1 / 2$ Heisenberg chain model are well described with the ansatz. The method is also applied to the two-dimensional $S=1 / 2$ Heisenberg and $\mathrm{U}(1)$ plaquette chain models.
\end{abstract}

RHIC experiments have started to test the fundamental properties of quantum chromodynamics (QCD). The importance of first-principle analysis of QCD has increased largely in the context of color confinement. There is the expectation that quarks and gluons deconfine in extreme conditions such as heavy ion collision. However, lattice gauge theory at finite density has been stuck for a long time. The most ideal treatment of nonequilibrium quantum physics is to trace time-evolution of quantum states based on the Schrödinger equation.

Density Matrix Renormalization Group (DMRG) is the variational method that gives the most accurate results in one-dimensional quantum systems [1]. In these days, DMRG has been used as a standard method to complement quantum Monte Carlo and successful in solving one-dimensional (zero and finite temperature) and two-dimensional (zero temperature) quantum systems. Application of DMRG to elemanrary particle and molecular physics has started some years ago. Recent interesting progress of DMRG is its application to non-equilibrium quantum physics and quantum information theory [2].

The first application of DMRG to particle physics was the massive Schwinger model with the $\theta$ term [3]. There is an old prediction by S. Coleman that quarks deconfine at $\theta=\pi[4]$. The model has not been analyzed accurately with Monte Carlo because of the sign problem. On the other hand, DMRG has been successful in describing the details of the phase transition with large lattices because DMRG is free from the sign problem.

The second application has been given by the author [5]. It is a preliminary work for study of gauge theory. In bosonic lattice systems, each site has infinite degrees of freedom and therefore hamiltonian is infinite dimensional differently from spin and fermion systems. It is not evident whether DMRG truncation works for bosonic degrees of freedom. DMRG needs to be tested in a simpler bosonic model before going to gauge theory. In Ref. [5], DMRG has been applied to a $(1+1)$-dimensional $\lambda \phi^{4}$ model. The DMRG result for the critical exponent $\beta=0.1264 \pm 0.0073$ is consistent with the exact one $\beta=1 / 8=0.125$. 
TABLE 1. Numerical results for ground-state energy per site in the $S=1 / 2$ Heisenberg chain model. The exact values have been obtained with the Bethe ansatz.

\begin{tabular}{rllll}
\hline$M \backslash L$ & 10 & 100 & 1000 & 10000 \\
\hline 6 & -0.4092 & -0.4372 & -0.4371 & -0.4368 \\
12 & -0.4092 & -0.4427 & -0.4425 & -0.4425 \\
Exact & -0.4515 & -0.4438 & -0.4431 & -0.4431 \\
\hline
\end{tabular}

Matrix product variational method is a result of large simplification of DMRG [6]. In this method, the energy function has a simple form and easy to evaluate. However, the advantage is lost if a constraint is imposed to variational space directly. The Gauss law in gauge theory is one of the most important examples of constraints. To avoid this difficulty, the author has developed a method to introduce constraints with undetermined multipliers in Hamiltonian [7]. Also, the author has generalized the matrix product variational method so that it can be applied to higher-dimensional general systems [8].

We introduce matrix product states according to Ref. [6]. With the knowledge from DMRG, wavefunction is represented as a product of matrices.

$$
|\Psi\rangle=\sum_{s_{1}, \ldots, s_{L}=1}^{K} \operatorname{tr}\left[A\left[s_{1}\right] \ldots A\left[s_{L}\right]\right]\left|s_{1}\right\rangle \ldots\left|s_{L}\right\rangle,
$$

where periodicity is assumed. $A[s]$ has the following normalization condition.

$$
\sum_{\beta=1}^{M} \sum_{s=1}^{K} A^{*}[s]_{\alpha \beta} A[s]_{\alpha^{\prime} \beta}=\delta_{\alpha \alpha^{\prime}}
$$

Each of the matrices $A[s]$ can be parameterized with an appropriate number of independent variables [7]. It is expected that better results is obtained for larger $M$. Let us apply the ansatz to the $S=1 / 2$ Heisenberg chain model with periodic boundary conditions

$$
H=\sum_{i=1}^{L}\left(S_{i}^{x} S_{i+1}^{x}+S_{i}^{y} S_{i+1}^{y}+S_{i}^{z} S_{i+1}^{z}\right)
$$

Periodicity simplifies calculation of the energy function

$$
E[A]=\frac{\langle\Psi|H| \Psi\rangle}{\langle\Psi \mid \Psi\rangle}=\sum_{a} \frac{\operatorname{tr}\left(\hat{S}^{a} \hat{S}^{a} \hat{1}^{L-2}\right)}{\operatorname{tr}\left(\hat{1}^{L}\right)},
$$

where $\hat{S}^{a}=\sum_{s, s^{\prime}}\left\langle s\left|S_{1}^{a}\right| s^{\prime}\right\rangle A^{*}[s] \otimes A\left[s^{\prime}\right]$ and $\hat{1}=\sum_{s} A^{*}[s] \otimes A[s]$. The minimum of the energy function corresponds to the ground state. In actual numerical calculation, the matrix $\hat{1}$ is diagonalized to simplify calculation of the powers of $\hat{1}$.

Table 1 shows numerical results for ground-state energy per site, which are compared with the exact ones. $M$ is the size of the matrices $A[s]$. When the lattice size $L$ is small, convergence is poor even for large $M$. On the other hand, when $L$ is large, the numerical 


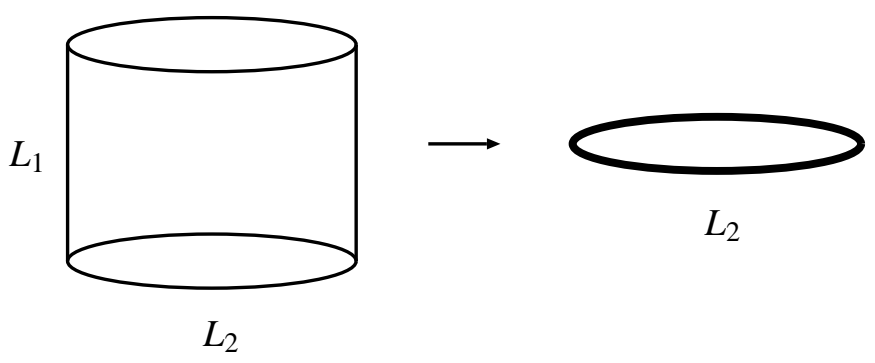

FIGURE 1. Reduction of two dimensional lattice to one dimensional

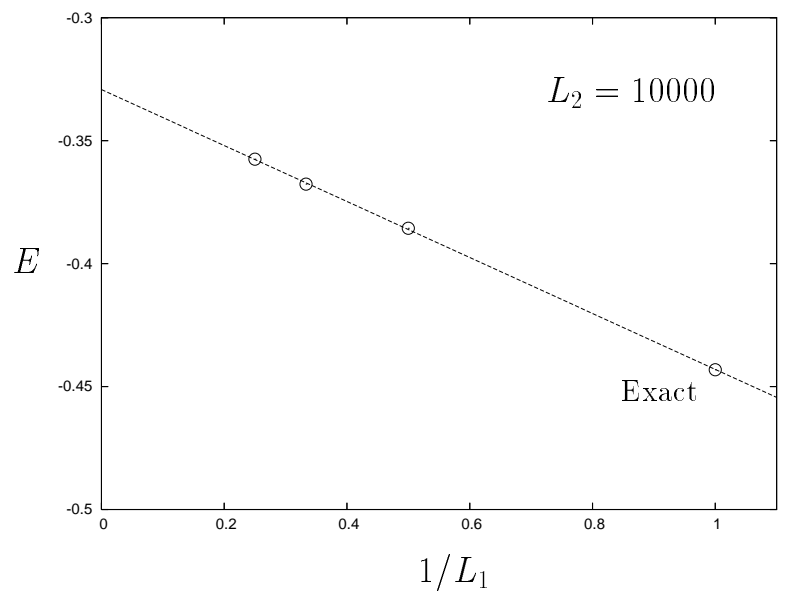

FIGURE 2. Energy per bond in the two-dimensional $S=1 / 2$ Heisenberg model.

results approaches the exact one as $M$ becomes larger. In the best case, error is less than $1 \%$. The lattice size dependence of energy is small when $L \geq 100$.

In the same way, the two-dimensional $S=1 / 2$ Heisenberg model is analyzed. To use the matrix product ansatz, one-dimensional structure needs to be found on the twodimensional lattice (see Fig. 1). The tube and ring sizes are denoted as $L_{1}$ and $L_{2}$, respectively. Figure 2 plots energy per bond as a function of $1 / L_{1}$ for a very large ring with $L_{2}=10000$. The circle for $L_{1}=1$ is the exact result from the Bethe ansatz. The other three circles have been calculated using the matrix product ansatz. We are going to make a comparison in the thermodynamic limit $L_{1} \rightarrow \infty$ by extrapolating the obtained points to the limit. In Table 2 , energy per bond is compared among various results in the thermodynamic result. "Lattice size" means the largest lattice size used for calculation.

The method is applied to a U(1) plaquette chain model. The hamiltonian is

$$
H=\sum_{l} E_{l}^{2}-x \sum_{\mathrm{p}}\left(U_{\mathrm{p}}+U_{\mathrm{p}}^{\dagger}\right)
$$

where $x \equiv 1 / g^{4}[13]$. Figure 3]plots energy as a function of the parameter $x$ and compares it with the results of Ref. [14]. In this calculation, the Gauss law $\nabla \cdot \mathbf{E}=0$ has not been imposed on the variational space. Since vacuum wavefunction is available, we can check gauge invariance of the obtained vacuum state by calculating the vacuum expectation value of the electric field. The obtained result $\sim 10^{-3}$ is small compared to energy, 
TABLE 2. Energy per bond of the two-dimensional $S=1 / 2$ Heisenberg model in the thermodynamic limit.

\begin{tabular}{lllll}
\hline Method & $E$ & Lattice size & Year & Reference \\
\hline Monte Carlo & -0.3347 & $16^{2}$ & 1999 & {$[9]$} \\
DMRG & -0.3347 & $12^{2}$ & 2001 & {$[10]$} \\
DMRG & -0.3321 & $20^{2}$ & 2003 & {$[11]$} \\
TPVA & -0.3272 & Very large & 2004 & {$[12]$} \\
Matrix product & $-0.3292 \pm 0.0005$ & $10000 \times 4$ & 2004 & This work \\
\hline
\end{tabular}

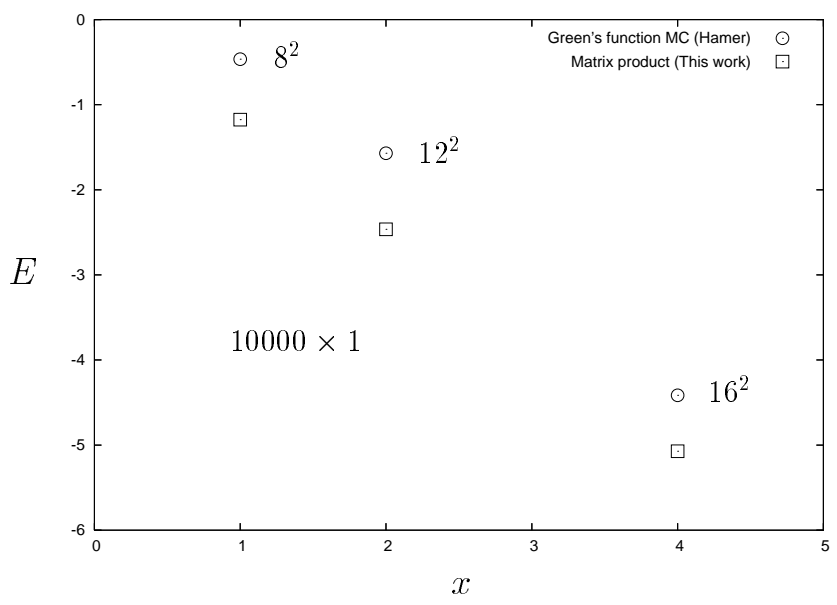

FIGURE 3. Energy per site vs $x$ in the U(1) hamiltonian lattice gauge model.

which shows approximate gauge invariance of the vacuum state. Currently, calculation with the Gauss law constraint is being conducted. Further precise analysis with larger lattices will be given elsewhere to refine the results shown in this presentation.

The numerical calculations were carried on the RIKEN RSCC system. This work has been partially supported by RIKEN BNL.

\section{REFERENCES}

1. S. R. White, Phys. Rev. Lett. 69, 2863 (1992); Phys. Rev. B 48, 10345 (1993).

2. U. Schöllwoeck, cond-mat/0409292

3. T. Byrnes, P. Sriganesh, R. J. Bursill, and C. J. Hamer, Phys. Rev. D 66, 013002 (2002).

4. S. R. Coleman, Annals Phys. 101, 239 (1976).

5. T. Sugihara, JHEP 0405, 007 (2004).

6. S. Östlund and S. Rommer, Phys. Rev. Lett. 75, 3537 (1995).

7. T. Sugihara, in preparation.

8. T. Sugihara, in preparation.

9. A. W. Sandvik, Phys. Rev. B 56, 678 (1997).

10. T. Xiang, J. Lou, and Z. Su, Phys. Rev. B 64, 104414 (2001).

11. D. J. J. Farnell, Phys. Rev. B 68, 134419 (2003).

12. Y. Nishio, N. Maeshima, A. Gendiar, and T. Nishino, cond-mat/0401115

13. J. B. Kogut and L. Susskind, Phys. Rev. D 11, 395 (1975).

14. C. J. Hamer, R. J. Bursill and M. Samaras, Phys. Rev. D 62, 054511 (2000). 